\title{
1 Population genomic evidence of selection on structural variants in a natural
}

2 hybrid zone

3

4 Linyi Zhang ${ }^{1,2, *}$, Samridhi Chaturvedi ${ }^{3}$, Chris C. Nice ${ }^{4}$, Lauren K. Lucas ${ }^{2}$, Zachariah Gompert ${ }^{2, \$}$

$5{ }^{1}$ Department of Ecology and Evolutionary Biology, University of Toronto, Toronto, Ontario,

6 Canada

$7 \quad{ }^{2}$ Department of Biology, Utah State University, Logan, UT 84322, USA

$8{ }^{3}$ Department of Integrative Biology, University of California, Berkeley, CA 94720, USA

$9 \quad{ }^{4}$ Department of Biology, Texas State University, San Marcos, TX 78666, USA

10

11 Address correspondence to:

12 *linyizhangecnu@gmail.com

13 \$zach.gompert@usu.edu 


\section{Abstract}

15 Structural variants (SVs) can promote speciation by directly causing reproductive isolation or by

16 suppressing recombination across large genomic regions. Whereas examples of each mechanism

17 have been documented, systematic tests of the role of SVs in speciation are lacking. Here, we take

18 advantage of long-read (Oxford nanopore) whole-genome sequencing and a hybrid zone between

19 two Lycaeides butterfly taxa (L. melissa and Jackson Hole Lycaeides) to comprehensively evaluate

20 genome-wide patterns of introgression for SVs and relate these patterns to hypotheses about

21 speciation. We found $>100,000$ SVs segregating within or between the two hybridizing species.

22 SVs and SNPs exhibited similar levels of genetic differentiation between species, with the

23 exception of inversions, which were more differentiated. We detected credible variation in patterns

24 of introgression among SV loci in the hybrid zone, with 562 of 1419 ancestry-informative SVs

25 exhibiting genomic clines that deviating from null expectations based on genome-average

26 ancestry. Overall, hybrids exhibited a directional shift towards Jackson Hole Lycaeides ancestry

27 at SV loci, consistent with the hypothesis that these loci experienced more selection on average

28 then SNP loci. Surprisingly, we found that deletions, rather than inversions, showed the highest

29 skew towards excess introgression from Jackson Hole Lycaeides. Excess Jackson Hole Lycaeides

30 ancestry in hybrids was also especially pronounced for Z-linked SVs and inversions containing

31 many genes. In conclusion, our results show that SVs are ubiquitous and suggest that SVs in

32 general, but especially deletions, might contribute disproportionately to hybrid fitness and thus

33 (partial) reproductive isolation. 


\section{Introduction}

Understanding the causes and evolution of species boundaries remains a central focus in evolutionary biology (Barton and Bengtsson 1986; Harrison and Larson 2014; Seehausen et al. 2014; Nosil et al. 2017, 2021). In particular, how species boundaries are maintained with ongoing gene flow is debated (Endler 1977; Felsenstein 1981; Servedio et al. 2011; Smadja and Butlin

412011 ; Nosil 2012). One possibility is that structural DNA changes, that is, genome rearrangements including deletions, duplications, insertions, translocations and inversions, promote speciation and maintain species boundaries despite opportunities for intermittent or ongoing gene flow (reviewed

44 in Wright 1978; Zhang et al. 2021a). Several mechanisms exist whereby structural changes to the genome might drive speciation. For example, structural genome rearrangements can cause meiosis to fail in hybrids leading to hybrid sterility, a common and effective barrier to gene flow (Stebbins 1958; Wright 1978; Coyne and Orr 2004; Zanders et al. 2014). Structural variants (SVs) can also directly cause reproductive isolation through their effects on traits, if, for example, insertions or deletions involve functionally important genes, or if the breakpoints of SVs disrupt a reading frame or alter gene expression (Lynch et al. 2001; Serrato-Capuchina and Matute 2018; Villoutreix et al.

51 2020; Zhang et al. 2021a). For instance, the insertion of a large transposable element altered the 52 expression of nearby genes in the peppered moth (Biston betularia), resulting in the well-known 53 melanic phenotype (Hof et al. 2016).

55 speciation with gene flow by inhibiting recombination within the inverted genomic region 56 (Hoffmann and Rieseberg 2008; Kirkpatrick 2010; Villoutreix et al. 2020; Zhang et al. 2021a). If

57 multiple mutations that contribute directly to reproductive isolation exist, are bought together, or 58 arise within the inverted genomic region, these barrier loci will be inherited as a unit (e.g., as a 
59 supergene) and protected from recombination making them more resistant to the homogenizing

60 effects of gene flow (Felsenstein 1981; Noor et al. 2001; Rieseberg 2001; Yeaman 2013). Large

61 inversions could be especially likely to contribute to reproductive isolation via suppressing

62 recombination because their size increases the chance that they will contain multiple, functionally

63 important genes, and thus that they will drive an increase in linkage disequilibrium among a set of

64 physically linked barrier loci. Many such inversion-associated supergenes have been suggested

65 with compelling evidence for a subset, but few have been clearly identified and linked directly to

66 reproductive isolation through their effect on recombination (but see Noor et al. 2001; Kozak et

67 al. 2017).

Indeed, there is increasing evidence of SVs associated with large phenotypic differences in

69 ecologically important traits, including those between ecotypes of sunflowers (Huang et al. 2020),

70 phenological shifts in Rhagoletis fruit flies (Feder et al. 2003), the repeated evolution of marine

71 and freshwater ecotypes of three-spined sticklebacks (Jones et al. 2012), and cryptic color pattern

72 variation in Timema stick insects (Villoutreix et al. 2020). However, not all of these differences

73 contribute to reproductive isolation (e.g., Comeault et al. 2015), nor is it always clear that the SV

74 mutations directly caused the trait differences. Thus, we lack a general understanding of how often

75 and in what ways SVs contribute to adaptation and speciation. One reason for this lingering

76 uncertainty is that most genomic studies of speciation rely on short-read next-generation

77 sequencing (NGS) (e.g., Illumina sequencing), which has a limited ability to identify and genotype

78 SVs, especially when SV breakpoints are located within repetitive regions in the genome

79 (Mahmoud et al. 2019; Ho et al. 2020). Recent advances in long-read sequencing technology, such

80 as Pacbio SMRT sequencing and Oxford Nanopore sequencing, which routinely produce 
81 sequencing fragments of $>10$ kilobases $(\mathrm{kbs})$, have enhanced our ability to detect SVs (van Dijk et

82 al. 2018; Amarasinghe et al. 2020).

Hybrid zones have long-been recognized as natural laboratories for studying speciation

84 (Hewitt 1988) and thus genomic analyses of hybrid zones with long-read DNA sequence data

85 could be especially informative about the contribution of SVs to speciation. Specifically, gene

86 flow and recombination in hybrid zones create combinations of parental alleles that are then tested

87 by selection under natural conditions (Rieseberg 2001; Gompert et al. 2012a; Schumer et al. 2018).

88 Patterns of ancestry and introgression in hybrid zones capture the outcome of these processes and

89 can be informative about the overall barrier to gene flow and the contribution of individual loci to

90 this barrier (Barton and Hewitt 1985; Barton and Gale 1993; Gompert et al. 2017). Consequently,

91 genomic studies of hybrid zones have contributed substantially to our knowledge of speciation, by

92 for example, providing evidence that sex chromosomes play a major role in speciation (Payseur et

93 al. 2004; Carling and Brumfield 2008; Chaturvedi et al. 2020) and by identifying genetic regions

94 with restricted or aberrant patterns of introgression suggestive of barrier loci (i.e., speciation genes)

95 (e.g., Teeter et al. 2010; Parchman et al. 2013; Knief et al. 2019; Wagner et al. 2020; Semenov et

96 al. 2021). Long-read DNA sequencing should allow more comprehensive and accurate genotyping

97 of SVs across hybrid zones (van Dijk et al. 2018; Amarasinghe et al. 2020), and thus make it

98 possible to study patterns of ancestry and introgression for SVs in hybrids at a genomic scale.

99 Doing so should allow us to better test long-standing theoretical predictions about the mechanisms

100 of SVs in promoting reproductive isolation (Weissensteiner et al. 2020). For example, we can ask

101 whether inversions show reduced or otherwise aberrant introgression compared to other loci

102 (including other types of SVs) as predicted from their role in recombination suppression, or

103 whether SVs in general show evidence of greater selection in hybrids than point mutations, as 
104 predicted if SVs often act as major effect mutations by deleting, disrupting or otherwise altering

105 genes and gene expression.

106 In this study, we used Oxford nanopore long-read DNA sequencing to analyze patterns of

107 introgression for SVs in a hybrid zone between two Lycaeides butterfly lineages. Lycaeides

108 consists of a complex of multiple species and hybrid lineages of small blue butterflies in North

109 America (Gompert et al. 2014). Hybridization between Lycaeides idas and L. melissa occurred in

110 the central Rocky Mountains 14,000 years ago (following the retreat of Pleistocene glaciers) and

111 has resulted in a series of admixed, partially stabilized populations in Jackson Hole valley and the

112 surrounding mountains (hereafter, Jackson Hole Lycaeides) (Gompert et al. 2010, 2012a, 2014).

113 In the last two hundred years, these ancient hybrids have secondarily hybridized with L. melissa,

114 creating a narrow (1-2 km) contemporary hybrid zone near Dubois, Wyoming, USA. In this study,

115 we focus on this contemporary hybrid zone. Previous work based on SNP data showed that these

116 contemporary hybrids exhibit a wide range of hybrid indexes but that they have, on average,

117 inherited more of their genomes from Jackson Hole Lycaeides than L. melissa, with a more

118 pronounced excess of Jackson Hole Lycaeides ancestry on the Z sex chromosome (Chaturvedi et

119 al. 2020).

120 In the present study, we first describe the number, size, type, and location of SVs across

121 the genome. We then quantify patterns of population genetic differentiation between L. melissa

122 and Jackson Hole Lycaeides at these SV loci. Next, we use genomic cline analysis to quantify

123 patterns of introgression within the Dubois hybrid zone for a set of 1419 ancestry informative SVs.

124 We then address the following questions based on analyses of these introgression patterns: 1) Is

125 there evidence that SVs experience more selection on average in hybrids than point mutations

126 (SNPs)? 2) Is the Z sex chromosome enriched for SV loci with patterns of introgression that deviate 
127 from null expectations (as was the case for SNPs in Chaturvedi et al. 2020)? 3) Do patterns of

128 introgression differ for different types of SVs, and do inversions specifically show the largest

129 deviations from null expectations? 4) Are patterns of introgression predicted by SV size or by the

130 number of genes within an SV? 5) How well does locus-specific differentiation between parental

131 taxa predict patterns of SV introgression in the hybrid zone?

132 Methods

133 Study system

134 Lycaeides idas and L. melissa diverged from an ancestral species that colonized North

135 America from Asia about 2.4 million years ago (Gompert et al. 2008; Vila et al. 2011). At present,

136 L. idas butterflies occur in northwestern North America including much of Canada, the

137 northwestern USA and the northern and central Rocky Mountains, whereas L. melissa are found

138 throughout the western USA. The two species' ranges thus overlap in parts of the Rocky Mountains

139 and northwestern USA (Scott 1992; Gompert et al. 2008). Lycaeides idas and L. melissa differ in

140 male genitalic morphology, wing pattern, host-plant use, and phenology (Gompert et al. 2010,

141 2013a,b; Lucas et al. 2018). Microhabitats where two species occur also tend to differ as L. melissa

142 more often occupy dry high desert, grassland, or agricultural areas, whereas L. idas are often found

143 in more mesic montane environments (Scott 1986). These two species came into secondary contact

144 in the central Rocky Mountains within the past 14,000 years where they hybridized resulting in a

145 broad, patchy hybrid zone comprising a series partially stabilized ancient, admixed populations in

146 the Jackson Hole valley, the Gros Ventre Mountains and the Yellowstone Plateau of northwestern

147 Wyoming (USA) (Gompert et al. 2010, 2012a, 2014). These admixed populations, (i.e., Jackson

148 Hole Lycaeides), carry more ancestry from L. idas than L. melissa, consistent with a tendency for

149 selection to favor L. idas alleles in the environment where these hybrids occur, which contains the 
150 same host plant used by nearby L. idas populations and is generally more L. idas-like (Gompert et

151 al. 2012a).

Within the past two hundred years, these ancient hybrid populations have come into contact

153 with and hybridized with L. melissa found on feral, roadside alfalfa (Medicago sativa) near the

154 town of Dubois, Wyoming (USA), creating a narrow (1-2 km) contemporary hybrid zone

155 (Chaturvedi et al. 2020). The hybrid zone lacks a clear spatial gradient instead comprising a single

156 admixed population with parents and many hybrids (Chaturvedi et al. 2020). In this study, we

157 analyze patterns of hybridization and introgression (i.e., the movement of alleles from one genomic

158 background to another) in this contemporary hybrid zone between Jackson Hole Lycaeides and $L$.

159 melissa. The high elevation (2115 meters), montane environment here is more similar to locations

160 where L. idas and Jackson Hole Lycaeides are found, whereas the immediate local habitat borders

161 agricultural fields similar to many L. melissa locations and the the main host plant here, alfalfa, is

162 used by L. melissa, but not L. idas or Jackson Hole Lycaeides (Gompert et al. 2013b; Chaturvedi

163 et al. 2020).

\section{DNA extraction, library preparation and MinION nanopore sequencing}

165 Genomic DNA from a total of 39 male butterflies (males are the homogametic sex),

166 including seven Jackson Hole Lycaeides, six L. melissa, and 26 Lycaeides from the Dubois hybrid

167 zone (Table S1) was isolated with Qiagen's MagAttract High-Molecular-Weight (HMW) DNA

168 extraction kit (cat. No. 67563; Qiagen Inc., Valencia, CA, USA). The concentration of DNA was

169 assessed using the dsDNA HS assay on a Qubit 4 fluorometer (Thermo Fisher).

170 We then created a DNA library for each butterfly for whole-genome long-read sequencing

171 on the Oxford MinION platform. Specifically, we first repaired DNA molecules with the NEBNext

172 FFPE DNA Repair Mix (NEB M6630) and NEBNext Ultra II end repair/dA-tailing Module (NEB 
173 E7546). This was done using 0.5-1.0 ug genomic DNA per sample and in accordance with the

174 manufacturer's protocol. Individuals with similar DNA concentrations were barcoded using the

175 Native Barcoding Expansion Pack 1-12 (EXP-NBD 104) and subsequently pooled to reach a total

176 amount of $1 \mu \mathrm{g}$ of genomic DNA or a total number of 12 individuals (whichever came first); the

177 number of individuals per pooled library was between 4 and 12 (Table S2). We then ligated adaptor

178 oligos for sequencing onto the barcoded fragments in each pooled library using the Ligation

179 Sequencing kit (SQK-LSK109). The ligation product from each library was then loaded onto R9.4

180 flow cell (FLO-Min106, ONT) for a sequencing run of a full 72 hours on a MinION. This generated

181 an average of 7 GB of sequence data per library, with a N50 read length of 1114 to 20,944 bps

182 (mean read length $=9073 \mathrm{bp}$; maximum read length of $342,840 \mathrm{bp}$ ).

183 Base calling, structural variant calling, and variant filtering

184 Base calling was performed using the ont-guppy algorithm from guppy_basecaller (version

185 4.2.2). We then used guppy_barcoder (version 4.2.2) to demultiplex the nanopore sequence reads

186 and subsequently remove the barcode sequences. Next, SVs, including inversions, tandem

187 duplications, insertions and deletions, relative to the newly updated L. melissa genome (see

188 supplemental material) were identified following Oxford Nanopore's suggested best practices (see

189 https://github.com/nanoporetech/pipeline-structural-variation) (Jain et al. 2018; Jiang et al. 2020;

190 Ren and Chaisson 2021). Here, a duplication is a specific type of insertion where the inserted

191 segment is a tandem repeat of the existing DNA region, and in general insertions versus deletions

192 are defined as added or missing DNA bases relative to the reference genome. Specifically, we used

193 Minimap2 (version 2.17) to align the nanopore reads to the reference genome with default

194 parameters including a mismatch penalty set at 4 and gap open penalty at 24 ( $\mathrm{Li} 2018)$. We then

195 used Sniffles (version 1.0.12) with default settings to identify SVs (Sedlazeck et al. 2018). This 
was done separately for each individual. Sniffles detects SVs from long-read alignments by using both within-alignment and split-read information, as small indels can be spanned within a single

198 alignment, whereas large SVs lead to split-read alignment (Sedlazeck et al., 2018). Importantly,

199 Sniffles filters false SV signals by considering both minimum read support as well as consistency

200 of the breakpoint position and size. Because loci with excessive htererozygosity are likely caused

201 by erroneous mapping of transposable elements to the reference genome (Jaegle et al. 2021), we

202 excluded SVs with excessive htererozygosity, defined for this purpose as all individuals in either

203 species initially being called heterozygotes because they had reads supporting the reference and

204 SV allele. Additionally, we excluded SVs with sequence data for less than $20 \%$ of the individuals,

205 which left a total of 290,276 SVs for downstream analysis. Next, we merged SVs across samples

206 using SURVIVOR (https://github.com/fritzsedlazeck/SURVIVOR; version 1.0.3) (Jeffares et al.

207 2017). When merging, we only considered SVs of at least 30 bps and merged those that overlapped

208 with a maximum allowed distance of $1 \mathrm{~kb}$ as measured pairwise between start and stop coordinates.

209 SURVIVOR does not compute genotype likelihoods, but rather only reports the number of

210 reads supporting the reference and alternative alleles and a called genotype based on a hard-

211 threshold number of reads (Jeffares et al. 2017). This ignores uncertainty in SV genotype. Thus,

212 rather than using the genotype calls, for each individual and locus we computed genotype

213 likelihoods for being homozygous for the reference allele, homozygous for the structural variant

214 allele or heterozygous. This allowed us to propagate uncertainty in structural variant genotypes to

215 downstream analyses (as is more routinely done with SNP data sets, e.g., Gompert et al. 2014).

216 Specifically, the genotype likelihoods were calculated based on the number of reads supporting

217 the reference and SV alleles and an assumed per-read SV error rate:

$$
L_{R A, R A}=(1-\varepsilon)^{n_{R E F}}(\varepsilon)^{n_{S V}}
$$

$$
L_{R A, S V}=(0.5)^{n_{R E F}} \quad(0.5)^{n_{S V}}
$$


Here, $L_{R A, R A}, L_{R A, S V}$ and $L_{S V, S V}$ refer to the relative likelihoods of being homozygous for the

222 reference allele, heterozygous and homozygous for the SV allele, respectively. The number of

223 reads supporting the reference and structural variant allele for an individual are denoted $n_{R E F}$ and

$224 n_{S V}$ and $\varepsilon$ refers to the error rate. This is not equivalent to the probability of a single base being

225 erroneous, but rather the probability of a sequence read erroneously supporting the reference or

226 structural variant allele. Because this error rate is not precisely known, we focus our main results

227 on the case of $\varepsilon=0.01$, but show results based on $\varepsilon=0.05$ in the online supplement (both gave

228 similar results; see the Supplemental Material for details).

229 With the computed genotype likelihood, allele frequencies for each SVs in L. melissa,

230 Jackson Hole Lycaeides and the Dubois hybrid zone were estimated using estpEM (version 0.1)

231 (Sorria-Carrasco et al. 2014; DRYAD https://doi.org/10.5061/dryad.nq67q) with a convergence

232 tolerance of 0.001 and $20 \mathrm{EM}$ iterations. The estpEM program infers allele frequencies via an

233 expectation-maximization (EM) algorithm while accounting for the uncertainty in genotypes (Li

234 2011). Only SVs with a minimum (global) minor allele frequency of 0.05 computed from all

235 individuals were retained. This gave us a total 127,574 SVs. For downstream population

236 introgression analyses (see below), we analyzed only SVs > 1000 bps in length, which left us with

23715,319 SVs.

$238 \quad$ Validating the structural variants with additional whole-genome sequencing

239 We generated additional whole-genome sequence data to validate the SVs from the 240 nanopore data. Specifically, we wanted to know whether a non-trivial subset of the SVs identified

241 by nanopore sequencing overlapped with those found by traditional, mate-pair Illumina

242 sequencing. For this, we used genomic data that were generated as part of a larger effort to produce

243 reference genomes for multiple Lycaeides species, but that have not been previously published. 
244 Thus, for this data set we focused on a smaller number of individuals, one from each of five

245 nominal lineages of Lycaeides in North America: L. idas (from Trout Lake, WY), L. melissa (from

246 Bonneville Shoreline Trail, UT), L. anna (from Yuba Gap, CA) and admixed lineages in the Sierra

247 Nevada (from Carson Pass, CA) and Warner mountains (from Buck Mountain, CA) in the western

248 USA. Genomic DNA was isolated from each butterfly using Qiagen's MatAttract HMW DNA

249 extraction kit (Qiagen, Inc.). We then outsourced library construction and sequencing to Macrogen

250 Inc. (Seoul, South Korea). A 3-kb mate-pair library was created for each butterfly species using

251 the Nextera Mate-pair Library Gel-plus kit (Illumina, Inc.). The libraries were then sequenced on

252 a HiSeq 2000 (2x100 bp reads) generating 669,157,572 Q30 reads (67,584,914,723 bps total or

$253 \sim 30 \mathrm{X}$ coverage per genome). SVs were identified by first using the SV callers DELLY and

254 LUMPY and then combining the output from these callers with SURVIVOR (version 1.0.7)

255 (Jeffares et al. 2017) (see the Supplemental Materials for details regarding DNA sequence

256 alignment, SV calling and filtering with the mate-pair data). After filtering, this left us with 12,265

257 SVs from the mate-pair data DNA sequence data set, including $3058 \mathrm{SVs}$ that were $>1 \mathrm{kbp}$ in

258 length.

259 Describing population genetic variation for structural variants in the nanopore data set

260 With allele frequency estimates using estpEM program, we computed pairwise FST among

261 L. melissa, Jackson Hole Lycaeides and the Dubois hybrid zone, with each of these treated as a

262 population. FST was calculated as $1 / L \sum_{L} \frac{\left(H|| T-H_{S}\right)}{1 / L \sum_{L} H_{T}}$, where $H_{S}$ and $H_{T}$ are the expected

263 subpopulation and total heterozygosities for each locus. We estimated Fst separately for each pair

264 of entities and SV type. Next, we designated a set of ancestry informative SVs as those having an

265 allele frequency difference $\geq 0.3$ between L. melissa and Jackson Hole Lycaeides, which we used

266 for all subsequent analyses. A total of 1419 SVs met this criterion. We used an admixture model, 
267 as implemented in Entropy (version 1.2), to estimate admixture proportions and genotypes for

268 these ancestry informative SVs (Gompert et al. 2014; Shastry et al. 2021). This model is similar to

269 the admixture model in STRUCTURE (Pritchard et al. 2000), except that the uncertainty in

270 genotypes due to limited sequencing coverage and errors are incorporated into the estimation of

271 genotypes and admixture proportions. We fit this model based on our computed genotype

272 likelihoods and assumed two source populations (i.e., $K=2$ as we had two hybridizing taxa). We

273 ran five Markov chain Monte Carlo (MCMC) chains with k=2, each with 10,000 MCMC iterations,

274 a burn-in of 5000 iterations and a thinning interval of 5. We obtained Bayesian genotype estimates

275 by taking the posterior mean of the number of $\mathrm{SV}$ alleles $(0,1$, or 2$)$ for each locus (this mean is

276 not constrained to be an integer value). Lastly, we visualized patterns of genetic variation at the

277 ancestry informative SVs using principal component analysis (PCA); this was done in R version

278 4.0.2 on the centered but not standardized genotype matrix.

\section{Estimating genomic cline parameters in the Dubois hybrid zone}

We quantified patterns of introgression for the SVs in the Dubois hybrid zone to test for

281 differential introgression of SVs relative to each other and relative to average introgression as

282 measured by previously published ancestry informative SNPs. We did this by fitting Bayesian

283 genomic clines with bgc (version 1.05a; https://github.com/zgompert/BGC-Bayesian-genomic-

284 clines) (Gompert and Buerkle 2011, 2012). Using genomic clines, it is possible to quantify

285 introgression into alternative genomic backgrounds even when hybrid zones do not display a

286 strong spatial axis (Gompert and Buerkle 2009, 2011), as is the case for the Dubois hybrid zone.

287 More specifically, deviations between genome-average introgression and the degree of

288 introgression for each SV locus were measured with two cline parameters, $\alpha$ and $\beta$. Cline parameter

$289 \alpha$ describes an overall deviation in the probability of ancestry relative to null expectations from an 
290 individual's hybrid index, where a positive (negative) value of $\alpha$ indicates an increase (decrease)

291 in the probability of reference species 1 ancestry (L. melissa ancestry in our analysis). Cline

292 parameter $\beta$ describes a deviation in the average rate of transition from reference species 0 ancestry

293 to reference species 1 ancestry along the genome-wide admixture gradient. Positive values for $\beta$

294 indicate a decrease in the rate of transition (i.e., alleles confined mostly to one genetic background),

295 whereas negative values for $\beta$ indicate a decrease in the rate of transition (i.e., alleles from both

296 species flowing more into alternative genomic backgrounds). Selection in hybrids can lead to

297 departures of cline parameters from genome-wide average admixture (Gompert et al. 2012b). For

298 example, selection generated by negative epistatic interactions (DMIs) can cause reduced

299 introgression (increased $\beta$ ) or a shift in ancestry towards one of the two species (high negative or

300 positive $\alpha$ ) (Gompert et al. 2012b). Drift can also cause deviations from genome-wide admixture,

301 but loci with credible deviations for $\alpha$ or $\beta$ should be enriched for loci affected (directly or

302 indirectly via LD) by selection (Gompert et al. 2012b). As this concerns selection in hybrids, this

303 includes possible barrier loci contributing to reproductive isolation.

In this study, we were interested in whether patterns of introgression for SVs differed

305 overall from genome-average admixture as measured by non-SV loci (i.e., SNPs). Therefore, we

306 used the SNP data to estimate hybrid index (i.e., admixture proportions) and thus the average extent

307 of introgression, and then fit genomic clines for the SV loci conditional on the SNP estimates of

308 hybrid index. The SNP data were taken from a previous study that included many of the individuals

309 used in this study (Chaturvedi et al. 2020) (see Table S2), with a total of 1164 ancestry-informative

310 markers (allele frequency differences between two parental species $\geq 0.3$ ). Clines were inferred

311 using a modified version of the bgc software that allows separate sets of loci to be used for fitting

312 clines and estimating hybrid indexes, and that does not require the cline parameters to sum to zero 
313 across loci (bgc version 1.05a). Thus, positive $\alpha$ values indicate an increase in the probability of

314 L. melissa ancestry relative to genome-wide average introgression based on SNPs (and vice a versa

315 for negative $\alpha$ ); positive $\beta$ for a structural variant locus would likewise indicate reduced

316 introgression compared to the average for the SNP loci. Genomic clines were fit using MCMC,

317 with five chains each with 200,000 iterations, a 20000-iteration burn-in, and a thinning interval of

318 5. We inspected the MCMC output to assess convergence of chains to the stationary distribution

319 and combined the output of the five chains. We designated SV loci as credibly deviating from

320 genome-average introgression when the $95 \%$ equal-tail probability intervals for $\alpha$ or $\beta$ excluded 0.

321 Five hundred sixty-two SVs exhibited credible deviation based on $\alpha$, but none did based on $\beta$; we

322 thus focus on $\alpha$ in the tests below.

We next conducted a series of statistical tests to analyze the estimated genomic clines with

324 respect to expectations from the hypothesis that SVs are a key driver of speciation. First, we tested

325 for an overall shift in the direction of introgression for SVs relative to SNPs by estimating the

326 mean value of the cline parameter $\alpha$ across SVs and by determining whether the observed

327 asymmetry in credible positive versus negative $\alpha$ values was consistent with binomial expectations

328 (with probability parameter $p=0.5$ ). Then, to determine whether patterns of introgression differed

329 between the autosomes and the Z-chromosome, we compared the mean signed $\alpha$ cline parameter

330 for Z-linked SVs to null expectations generated by randomly sampling the same number of SV

331 loci from the autosomes; this was done 1000 times. Next, to test whether patterns of introgression

332 differed for different types of SVs, and specifically whether inversions showed the largest

333 deviations from null expectations, we compared the mean value of signed $\alpha$ for inversions to null

334 expectations generated by a randomly sampling a subset of the same number of loci from all other

335 SV types (this was also done 1000 times). 
We then tested the hypothesis that SV length or the number of genes in a SV affects patterns of genetic differentiation between species and patterns of introgression in the hybrid zone (gene numbers were taken from the genome annotation described in Chaturvedi et al. 2020 and then transferred to our updated L. melissa genome; see the Supplemental Materials for details). As expected, SV length and number of genes within a SV were positively correlated (Pearson $\mathrm{r}=$ 0.863, 95\% CI: 0.862-0.865, $\mathrm{P}<0.001)$, and thus we fit multiple regression models with both covariates simultaneously to better assess their marginal effects. Specifically, we fit linear models

343 for $\alpha$ (one of the cline parameters) or the absolute value of $\alpha$ as a function of SV length (in bps),

344 number of annotated genes in each SV, and their interaction for each class of SVs (covariates were

345 centered and standardized prior to analysis). This was done with the $\mathrm{lm}$ function in $\mathrm{R}$ version 4.0.2.

346 Here, models for signed values of $\alpha$ detect factors associated with an overall directional shift (for

347 or against Jackson Hole or L. melissa ancestry), whereas models for the absolute value of $\alpha$ detect

348 factors associated with the magnitude of introgression independent of the direction. We fit similar 349 multiple regression models for the 1419 ancestry informative SVs for genetic differentiation (FST, 350 logit transformed) between the parental lineages as well.

Finally, we tested for an association between genetic differentiation at individual SV loci

352 between the allopatric Jackson Hole Lycaeides and L. melissa populations and patterns of 353 introgression for these loci in the hybrid zone. A positive association between FST and the absolute 354 value of $\alpha$ would be expected if differentiation in allopatry was driven by divergent selection and 355 the same SV loci under divergent selection in allopatry also affected hybrid fitness in the Dubois 356 hybrid zone (e.g., Gompert et al. 2012b). Moreover, a correlation between FST and the signed 357 (directional) value of $\alpha$ might be expected if alleles from one parental species are 358 disproportionately favored in the hybrid zone, either because of environment-dependent fitness or 
because of DMIs causing selection against minor parent ancestry (e.g., Schummer et al. 2018;

360 Chaturvedi et al. 2020). Thus, Pearson correlation tests were conducted for FST versus both

361 absolute $\alpha$ and signed $\alpha$ (this was done in $\mathrm{R}$ version 4.0.2). Importantly, whereas ancestry

362 informativeness can create spurious correlations between FST and cline parameters, past work

363 suggests this is unlikely when considering only ancestry informative markers as was done here

364 (Gompert et al. 2012b).

365 Results

Number, length, and distribution of SVs across genome

Using long-read nanopore sequencing, we identified a total of $127,574 \mathrm{SVs}$ that covered were 68,927 deletions covering $18.4 \%$ of the genome, 56,527 insertions covering $4.0 \%$ of the genome, 1503 inversions covering $7.2 \%$ of the genome and 617 duplications covering $0.7 \%$ of the genome (Figure 1A,B; see Table 1 and Figure 2A for range of SV sizes). Considering only SVs > $3721 \mathrm{kbp}$ in length, we identified 13,359 deletions covering $15.8 \%$ of the genome, 1073 inversions 373 covering $7.1 \%$ of the genome, 771 insertions covering $1.3 \%$ of the genome and 116 duplications 374 covering $0.1 \%$ of the genome (Figure 1C, 1D; Table 1).

We detected 12,265 SVs using mate-pair sequencing, 32.5\% of which corresponded with

376 SVs identified from nanopore sequencing (Table 2). Similar to the nanopore data, most of the SVs

377 detected from the mate-pair data were deletions (11,594 out of 12,265), followed by 224 378 duplications, and 156 inversions (Figure S2, S3).

\section{Population genomic patterns of structural variation}

The distribution of allele frequencies was skewed towards rare alleles among Lycaeides

381 individuals (Figure 2B), which is consistent with recent studies in grapevine, Drosophila, and 
songbirds SV (Chakraborty et al. 2019; Zhou et al. 2019; Weissensteiner et al. 2020). Most SVs exhibited only subtle allele frequency differences between L. melissa and Jackson Hole Lycaeides

384 (mean difference $=0.118$ ), but 1419 SVs exhibited allele frequency differences exceeding 0.3 , and

385 four SVs (all deletions) were fixed or nearly fixed between these taxa (i.e., allele frequency difference 1.0) (Figure S1). We detected modest differentiation between Jackson Hole Lycaeides and L. melissa based on the full set of 127,574 SVs $\left(\mathrm{F}_{\mathrm{ST}}=0.048\right)$ (Table 3). This is similar to an earlier estimate of genetic differentiation between these lineages based on SNP data $\left(\mathrm{F}_{\mathrm{ST}}=0.050\right)$ (Chaturvedi et al. 2020). However, genetic differentiation ( $\left.F_{S T}\right)$ for inversions was higher than for other SV types or SNPs $\left(F_{S T}=0.070\right)$ (Table 3$)$. Lastly, genetic differentiation was positively associated with deletion length and the number of genes in a deletion (with a positive interaction between these two covariates) (Table 4), but no such associations were detected for the other SV 393 types. generally similar patterns of genetic variation in the parental lineages and hybrids as past work using SNPs (e.g., Chaturvedi et al. 2020) (Figure 3). For example, genetic PC scores from SNPs and SVs were highly positively correlated (Pearson $r=0.772, P<0.0001$ ) and Lycaeides from the Dubois hybrid zone displayed a range of admixture proportions (i.e., hybrid indexes) from nearly pure Jackson Hole Lycaeides to L. melissa with many intermediates (Figure 3B, 3C).

We observed qualitative variation in patterns of introgression in the hybrid zone for the 1419 SVs (Figure 4), which was confirmed quantitatively using genomic cline analysis (Figure 5).

403 Specifically, credible variation in patterns of introgression across the genome were detected for 404562 SVs (cases where 95\% equal-tail probability intervals [ETPIs] for genomic cline parameters 
did not overlap with zero) (Figure 5B, C). Relative to genome-average introgression computed

406 from ancestry-informative SNPs, a total of $152 \mathrm{SVs}$ showed credible excess introgression from $L$.

407 melissa (95\% ETPIs for cline parameter $\alpha>0)$ and 410 SVs exhibited credible excess introgression

408 from Jackson Hole Lycaeides (95\% ETPIs for cline parameter $\alpha<0$ ). Consequently, relative to

409 average introgression for SNPs, SVs showed an excess of Jackson Hole Lycaeides ancestry in the

410 hybrid zone (mean $\alpha \pm \mathrm{SE}=-0.295 \pm 0.018$; probability of getting 410 credible excessive Jackson

411 Hole ancestry loci out of 562 credible excess introgress loci: $P<0.0001$, Figure 5C). No SVs

412 displayed credible shifts in the rate or extent of introgression (95\% ETPIs for cline parameter $\beta>$

4130 or $\beta<0$ ) (detecting credible deviations based on beta often requires larger sample sizes across

414 the range of hybrid indexes).

415 Structural variants exhibiting excess directional introgression were distributed across the

41623 Lycaeides chromosomes, but SVs on the Z-chromosome showed significantly more excess

417 Jackson Hole Lycaeides introgression compared to SVs on the autosomes (permutation test with

4181000 permutations: mean difference in signed $\alpha[\mathrm{Z}$ - autosomes $]=-0.268, P<0.001)$. The degree

419 of directional introgression also differed among different types of SVs, with deletions showing

420 significantly more excess Jackson Hole Lycaeides introgression than inversions (permutation test

421 with 1000 permutations: mean differences in signed $\alpha$ [deletion - inversion] $=-0.232, P<0.001$,

422 Figure 6A).

423 We did not detect significant associations of SV length or the number of genes in SVs with

424 the absolute value of $\alpha$ (i.e., the extent of excess introgression regardless of direction) for any of

425 the SV types based on the multiple regression analyses (Table S3). However, the degree of

426 directional introgression $(\operatorname{signed} \alpha)$ was positively associated with the number of genes contained

427 (regression coefficient $=0.19, t=2.35, P=0.021$ ) but not SV length for inversions (regression 
coefficient $\approx 0, t=-0.79, P=0.431$; Table 5 and Figure $6 \mathrm{~B})$. In contrast, the degree of directional

429 introgression ( signed $\alpha$ ) was positively associated with the length (regression coefficient $=0.248$,

$430 t=3.02, P=0.003$ ) but not the number of genes contained within deletions (regression coefficient

$431=0.02, t=0.15, P=0.883$; Table 5).

432 Finally, genetic differentiation ( $\mathrm{F}_{\mathrm{ST}}$ ) between the Lycaeides melissa and Jackson Hole

433 Lycaeides was moderately predictive of patterns of introgression in the hybrid zone. Specifically,

434 FST was positively associated with the absolute value of $\alpha$ (Pearson $r=0.171,95 \% \mathrm{CI}=0.120$ -

$4350.222, P<0.001)$ and negatively associated with signed values of $\alpha$ (Pearson $r=-0.160,95 \% \mathrm{CI}$ :

$436-0.210--0.108, P<0.001)$. Thus, SVs that differed more in frequency between the parental

437 lineages exhibited more pronounced excess introgression overall, along with a specific shift

438 towards excess Jackson Hole Lycaeides ancestry in the hybrid zone.

\section{Discussion} genes or mutations causing reproductive isolation and determining how often particular types of

442 genetic change are involved (Serrato-Capuchina and Matute 2018). Hybrid zones provide a

443 powerful tool to address both of these goals (Fitzpatrick et al. 2010; Harrison and Larson 2014;

444 Gompert et al. 2017). First, quantifying variability in patterns of introgression across the genome

445 in hybrid zones can lead to the identification of genomic regions or genes causing reproductive

446 isolation in nature (Gompert and Buerkle 2009; Payseur 2010). Second, contrasting patterns of

447 introgression across different categories of genetic or structural variants can help determine

448 whether certain types of changes contribute disproportionately to speciation. Several models of

449 speciation suggest a prominent role for SVs, including models of DMIs evolving following gene

450 duplication, models involving recombination suppression via inversions, and various models of 
451 chromosomal speciation (e.g., Wright 1978; Lynch and Force 2000; Lynch et al. 2001; Feder and

452 Nosil 2009; Zuellig and Sweigart 2018). Nonetheless, genome-wide characterization of patterns

453 of structural variation needed to systematically address these hypotheses have been mostly lacking.

454 We overcame this constraint using long-read Oxford nanopore sequencing and thereby quantified

455 genome-wide patterns of introgression for SVs. We discuss technical aspects of our findings and

456 the implications of our findings for understanding speciation below.

\section{Prevalence of SVs and sequencing technologies}

We found that SVs are prevalent in Lycaeides butterflies, covering about $30 \%$ of the genome overall (though not $30 \%$ in any one individual). Considerably fewer SVs were detected using standard mate-pair sequencing (127,574 for nanopore vs 11,974 for mate-pair sequencing), but many that were found with mate-pair sequencing coincided with SVs from the nanopore data set (3891 out of 11974 SVs, 32\%) (note that the two datasets also differed in the sampling scheme).

463 This both bolsters our confidence in the nanopore data and highlights the utility of nanopore 464 sequencing for SV genotyping relative to mate-pair sequencing. An additional $3842 \mathrm{SVs}$ from the 465 mate-pair data set were initially included in the nanopore data set but were removed during filtering 466 because all individuals from one of the two parental species were heterozygotes. Most of the SVs 467 with this pattern of excessive heterozygosity were deletions or insertions, consistent with 468 expectations for pseudo-heterozygosity caused by errors in mapping transposable elements to the 469 reference genome (Jaegle et al. 2021). The proportion of SVs exhibiting excessive heterozygosity 470 from the mate-pair sequencing data set $(\sim 32 \%)$ was much higher than for the nanopore sequence 471 data set $(\sim 12.7 \%)$. This discrepancy likely reflects the fact that short-read sequencing is unable to 472 accurately capture the breakpoints of SVs that are located within repetitive regions of the genome. 
473 Thus, our results further highlight the advantages of applying long-read sequencing for identifying

474 SVs.

475 Structural variants, introgression and speciation

476 Consistent with past work (Chaturvedi et al., 2020), we found evidence that patterns of

477 introgression varied across the genome, with some SVs showing excess Jackson Hole Lycaeides

478 ancestry and some showing excess L. melissa ancestry. Moreover, across SVs there was an overall

479 excess of loci with excess Jackson Hole Lycaeides ancestry relative to genome-average

480 introgression based on SNP loci. Such a pattern would be expected if (i) selection in the hybrid

481 zone, when it occurs, more frequently favors Jackson Hole Lycaeides alleles than L. melissa

482 alleles, and if (ii) a greater proportion of SV loci are subject to selection than SNP loci, including

483 direct and indirect selection caused by LD with actual barrier loci. Selection for Jackson Hole

484 Lycaeides alleles could be driven by the environment, as the hybrid zone habitat is generally more

485 like that found in nearby Jackson Hole populations, including a short summer season that is

486 associated with univoltinism and obligate diapause as observed in L. idas and Jackson Hole

487 Lycaeides, but not L. melissa (in contrast, the immediate agricultural habitat and host plant are

488 indicative of L. melissa) (Gompert et al. 2013b). However, this result is also consistent with DMIs

489 causing selection against minor-parent alleles (i.e., alleles from the parent species that has

490 contributed less overall to an admixed population or hybrid zone) (Schumer et al. 2018; Martin et

491 al. 2019; Chaturvedi et al. 2020). Lastly, because Jackson Hole Lycaeides are themselves ancient

492 hybrids with L. melissa as one parent, their genomes might be enriched for alleles that function

493 well in hybrids and thus are less likely to be selected against in a contemporary hybrid zone.

494 Finally, the pattern that SVs show more evidence suggestive of selection than SNPs is consistent 
with recent studies of SVs in other organisms, including Drosophila (Weissensteiner et al. 2020) and grapevine (Zhou 2019).

Also consistent with past work (Chaturvedi et al., 2020), the Z sex chromosome was 498 enriched for SV loci with patterns of introgression that deviated from the genome-wide average.

499 This supports theory and empirical studies, which have found sex chromosomes plays a 500 disproportional role in determining hybrid fitness (Coyne 1985; Masly and Presgraves 2007; 501 Payseur and Rieseberg 2016; Gompert et al. 2017). The overall shift towards excess Jackson Hole 502 Lycaeides ancestry noted above was also especially pronounced on the $\mathrm{Z}$ chromosome, which again could be explained by environment-dependent selection or an excess contribution to DMIs. maintaining and promoting speciation (Noor et al. 2001; Rieseberg 2001; Hooper et al. 2019) (reviewed by Faria and Navarro 2010; Zhang et al. 2021a). We found little support for this 507 hypothesis in Lycaeides butterflies Specifically, although inversions were more genetically 508 differentiated between allopatric Jackson Hole Lycaeides and L. melissa populations, this 509 increased differentiation did not result in exceptional patterns of introgression in the hybrid zone 510 as would be expected if these loci were enriched for barrier loci. We did find an alternative, and 511 somewhat unexpected pattern of introgression for inversions; signed estimates of $\alpha$ exhibited a 512 modest, positive correlation with inversions containing more genes (Table 5, Figure 6B). In other 513 words, in contrast to an overall signal of excess Jackson Hole ancestry in the hybrid zone relative 514 to average ancestry based on SNPs, we detected excess L. melissa ancestry for inversions 515 containing many genes. Because the hybrid zone has more individuals with Jackson Hole 516 Lycaeides ancestry overall (Chaturvedi et al. 2020), this means that large inversions carrying 517 excess L. melissa ancestry should frequently occur in a heterozygous state. One possible 
518 explanation for this pattern is that inversions with many genes contain a greater number of

519 recessive deleterious mutations with different mutations associated with different inversion alleles,

520 such that inversion heterozygotes would be heterozygous for these mutations and thus gain a

521 fitness advantage via associative overdominance (Ohta 1971; Faria et al. 2019; Jay et al. 2021).

522 Such an explanation was recently invoked to explain inversion polymorphisms among mimicry

523 supergenes in Heliconius butterflies (Jay et al., 2021). More generally, empirical support from

524 genomic studies for inversions contributing disproportionately to speciation has been mixed, with

525 some studies finding no evidence that inversions play a major role in maintaining species

526 boundaries, including sympatric Heliconius butterfly species (Davey et al. 2017) and Timema

527 walking sticks (Lucek et al. 2019).

528 In contrast, deletions, which were the most common SV identified, exhibited the greatest

529 overall deviations from genome-average patterns of introgression (507 of 562 SVs that deviated

530 from null expectations of introgression were deletions). Moreover, ancestry informative deletions

531 contain more genes than non-ancestry informative deletions (1000 permutation test: $p<0.001$; for

532 a list the 169 annotated genes that are wholly or partially deleted by ancestry-informative deletions

533 see Table S4). Some deletions might associate with phenotypic changes. For instance, one deletion

534 showed excessive Jackson Hole Lycaeides ancestry contained gene Fry that could affect

535 morphogenesis. Patterns of deletions are consistent with these loci being enriched for genetic

536 variants contributing to reproductive isolation or those in linkage disequilibrium with such

537 variants. Large deletions could generate underdominance of heterozygotes when potential

538 functional genes are deleted or gene duplication followed by deletions, causing DMI (see table S4,

539 Force et al. 1999; Lynch and Force 2000). 
Finally, we found evidence that SVs that differed more between L. melissa and Jackson

541 Hole Lycaeides exhibited excess directional introgression in Dubois, and specifically an excess of

542 Jackson Hole Lycaeides ancestry. Still, genetic differentiation (FST) only explained 3\% (squared

543 correlation) of the variation in $\alpha$. Particularly, inversions exhibit the highest FST between parental

544 species compared to other SVs, but the lowest degree of deviation in introgression pattern. Taken

545 together, these patterns highlighting the fact that differentiation in allopatry is far from a perfect

546 predictor of introgression in hybrid zones and thus only somewhat informative of a genetic

547 region's contribution to speciation (Cruickshank and Hahn 2014; Burri 2017). This imperfect

548 prediction of loci underlying genetic differentiation between L. melissa and Jackson Hole

549 Lycaeides as barrier loci is consistent with previous study in hybrid zone between L. melissa and

550 L. idas (Gompert et al. 2012a). The disconnection between loci underlying genetic differentiation

551 and barrier loci could due to the limited genetic differentiation $\left(\mathrm{F}_{\mathrm{ST}}=0.05\right)$ where differentiated

552 loci are less likely cause DMI among hybrids, or because of the environmental dependent hybrid

553 fitness (Zhang et al. 2021b; Thompson et al. 2022). Altogether, our finding reveals the complexity

554 of predicting the genetic basis of hybrid fitness based on parental differentiation.

556 Acknowledgments

557 Funding for this project was provided by US NSF grants to ZG (1844941) and CCN (1050355).

558 The support and resources from the Center for High Performance Computing at the University of 559 Utah are gratefully acknowledged. 
562 Raw nanopore DNA sequence data will be deposited in the NCBI SRA (BioProject \# pending).

563 Mate-pair DNA sequence will be deposited in the NCBI SRA (BioProject \# pending). Our new $L$.

564 melissa genome assembly will be deposited in the NCBI Reference Sequence Database (accession

565 number pending). Computer code and scripts central to this manuscript are available from GitHub:

566 source code for genomic cline analysis (i.e., bgc) https://github.com/zgompert/BGC-Bayesian-

567 genomic-clines, scripts for processing and analyzing the nanopore data

568 (https://github.com/lz41/2022-Structural-variants), scripts for processing and analyzing the mate-

569 pair DNA sequence data (https://github.com/lz41/2022-Structural-variants). Benefits from this

570 research accrue from the sharing of our data and results on public databases as described above.

571 Author Contributions

$572 \mathrm{LZ}$ and ZG designed the research. LZ, SC, CCN, LKL and ZG performed the research. LZ and

573 SC analyzed the data. LZ wrote the paper. LZ, SC, CCN, LKL and ZG edited and revised the

574 paper.

\section{References}

577 Amarasinghe, S. L., S. Su, X. Dong, L. Zappia, M. E. Ritchie, and Q. Gouil. 2020. Opportunities and challenges in long-read sequencing data analysis. Genome Biology 21:30.

Barton, N., and B. O. Bengtsson. 1986. The barrier to genetic exchange between hybridising populations. Heredity 57:357-376.

Barton, N. H. \& Gale, K. S. 1993 Genetic analysis of hybrid zones. In Hybrid zones and the evolutionary process (ed. R. G. Harrison), pp. 13-45. New York, NY: Oxford University Press

Barton, N. H., and G. M. Hewitt. 1985. Analysis of Hybrid Zones. Annual Review of Ecology and Systematics 16:113-148.

Burri, R. 2017. Interpreting differentiation landscapes in the light of long-term linked selection. Evolution Letters 1:118-131. 
Carling, M. D., and R. T. Brumfield. 2008. Haldane's rule in an avian system: using cline theory and divergence population genetics to test for differential introgression of mitochondrial, autosomal, and sex-linked loci across the Passerina bunting hybrid zone. Evolution 62:2600-2615.

Chaturvedi, S., L. K. Lucas, C. A. Buerkle, J. A. Fordyce, M. L. Forister, C. C. Nice, and Z. Gompert. 2020. Recent hybrids recapitulate ancient hybrid outcomes. Nature Communication 11:2179.

Comeault, A. A., S. M. Flaxman, R. Riesch, E. Curran, V. Soria-Carrasco, Z. Gompert, T. E.

Coyne, J. A. 1985. The genetic basis of Haldane's rule. Nature 314:736-738.

Coyne, J. A., and H. A. Orr. 2004. Speciation. Sinauer Associates Sunderland, MA.

Cruickshank, T. E., and M. W. Hahn. 2014. Reanalysis suggests that genomic islands of speciation are due to reduced diversity, not reduced gene flow. Molecular Ecology 23:3133-3157.

Davey, J. W., S. L. Barker, P. M. Rastas, A. Pinharanda, S. H. Martin, R. Durbin, W. O. McMillan, R. M. Merrill, and C. D. Jiggins. 2017. No evidence for maintenance of a sympatric Heliconius species barrier by chromosomal inversions. Evolution Letters $1: 138-154$.

Endler, J. A. 2020. Geographic Variation, Speciation and Clines. (MPB-10), Volume 10. Princeton University Press.

610 Faria, R., and A. Navarro. 2010. Chromosomal speciation revisited: rearranging theory with pieces of evidence. Trends in Ecology \& Evolution 25:660-669.

612 Faria, R., K. Johannesson, R. K. Butlin, and A. M. Westram. 2019. Evolving inversions. Trends in ecology \& evolution 34(3), 239-248.

614 Feder, J. L., S. H. Berlocher, J. B. Roethele, H. Dambroski, J. J. Smith, W. L. Perry, V. Gavrilovic, K. E. Filchak, J. Rull, and M. Aluja. 2003. Allopatric genetic origins for sympatric host-plant shifts and race formation in Rhagoletis. Proceedings of National Academy of Sciences of the United States of America 100:10314-10319. 
618 Feder, J. L., and P. Nosil. 2009. Chromosomal inversions and species differences: when are genes affecting adaptive divergence and reproductive isolation expected to reside within inversions? Evolution 63:3061-3075.

Felsenstein, J. 1981. Skepticism towards santa rosalia, or why are there so few kinds of animals? Evolution 35:124-138.

Fitzpatrick, B. M., J. R. Johnson, D. K. Kump, J. J. Smith, S. R. Voss, and H. B. Shaffer. 2010. Rapid spread of invasive genes into a threatened native species. Proceedings of National Academy of Sciences of the United States of America 107:3606-3610.

Gompert, Z., and C. A. Buerkle. 2009. A powerful regression-based method for admixture mapping of isolation across the genome of hybrids. Molecular Ecology 18:1207-1224.

Gompert, Z., and C. A. Buerkle. 2011. Bayesian estimation of genomic clines. Molecular Ecology 20:2111-2127.

Gompert, Z., and C. A. Buerkle. 2012. bgc: Software for Bayesian estimation of genomic clines. Molecular Ecology Resources 12:1168-1176.

Gompert, Z., L. K. Lucas, C. A. Buerkle, M. L. Forister, J. A. Fordyce, and C. C. Nice. 2014.

Gompert, Z., M. L. Forister, J. A. Fordyce, and C. C. Nice. 2008. Widespread mito-nuclear discordance with evidence for introgressive hybridization and selective sweeps in Lycaeides. Molecular Ecology 17:5231-5244. Admixture and the organization of genetic diversity in a butterfly species complex revealed through common and rare genetic variants. Molecular Ecology 23:4555-4573.

644 Gompert, Z., L. K. Lucas, C. C. Nice, J. A. Fordyce, C. Alex Buerkle, and M. L. Forister. 2013 b. 
Gompert, Z., L. K. Lucas, C. C. Nice, J. A. Fordyce, M. L. Forister, and C. A. Buerkle. 2012 a. Genomic regions with a history of divergent selection affect fitness of hybrids between two butterfly species. Evolution 66:2167-2181.

Gompert, Z., E. G. Mandeville, and C. A. Buerkle. 2017. Analysis of population genomic data from hybrid zones. Annual Review of Ecology, Evolution, and Systematics 48:207-229.

Gompert, Z., T. L. Parchman, and C. A. Buerkle. 2012b. Genomics of isolation in hybrids. Philosophical Transactions of the Royal Society B: Biological Sciences 367:439-450.

Harrison, R. G., and E. L. Larson. 2014. Hybridization, introgression, and the nature of species boundaries. Journal of Heredity 105:795-809.

Hewitt, G. M. 1988. Hybrid zones-natural laboratories for evolutionary studies. Trends in Ecology \& Evolution 3:158-167.

Ho, S. S., A. E. Urban, and R. E. Mills. 2020. Structural variation in the sequencing era. Nature Review in Genetics 21:171-189.

Hof, A. E. van't, P. Campagne, D. J. Rigden, C. J. Yung, J. Lingley, M. A. Quail, N. Hall, A. C. Darby, and I. J. Saccheri. 2016. The industrial melanism mutation in British peppered moths is a transposable element. Nature 534:102-105.

Hoffmann, A. A., and L. H. Rieseberg. 2008. Revisiting the impact of inversions in evolution: from population genetic markers to drivers of adaptive shifts and speciation? Annual Reviews in Ecology, Evolution, and Systematics 39:21-42.

Hooper, D. M., S. C. Griffith, and T. D. Price. 2019. Sex chromosome inversions enforce reproductive isolation across an avian hybrid zone. Molecular Ecology 28:1246-1262.

Huang, K., R. L. Andrew, G. L. Owens, K. L. Ostevik, and L. H. Rieseberg. 2020. Multiple

Jaegle, B., L. M. Soto-Jiménez, R. Burns, F. A. Rabanal, and M. Nordborg. 2021. Extensive T. Dilthey, I. T. Fiddes, S. Malla, H. Marriott, T. Nieto, J. O’Grady, H. E. Olsen, B. S. Pedersen, A. Rhie, H. Richardson, A. R. Quinlan, T. P. Snutch, L. Tee, B. Paten, A. M. 
Phillippy, J. T. Simpson, N. J. Loman, and M. Loose. 2018. Nanopore sequencing and assembly of a human genome with ultra-long reads. Nature Biotechnology 36:338-345.

Jay, P., M. Chouteau, A. Whibley, H. Bastide, H. Parrinello, V. Llaurens, and M. Joron. 2021. Mutation load at a mimicry supergene sheds new light on the evolution of inversion polymorphisms. Nature Genetics 53:288-293.

Jeffares, D. C., C. Jolly, M. Hoti, D. Speed, L. Shaw, C. Rallis, F. Balloux, C. Dessimoz, J. Bähler, and F. J. Sedlazeck. 2017. Transient structural variations have strong effects on quantitative traits and reproductive isolation in fission yeast. Nature Communications 8:14061.

Jiang, T., Y. Liu, Y. Jiang, J. Li, Y. Gao, Z. Cui, Y. Liu, B. Liu, and Y. Wang. 2020. Long-read-

Jones, F. C., M. G. Grabherr, Y. F. Chan, P. Russell, E. Mauceli, J. Johnson, R. Swofford, M.

Kirkpatrick, M. 2010. How and Why Chromosome Inversions Evolve. PLOS Biology 8:e1000501.

Knief, U., C. M. Bossu, N. Saino, B. Hansson, J. Poelstra, N. Vijay, M. Weissensteiner, and J. B. W. Wolf. 2019. Epistatic mutations under divergent selection govern phenotypic variation in the crow hybrid zone. Nature Ecology and Evolution 3:570-576.

Kozak, G. M., C. B. Wadsworth, S. C. Kahne, S. M. Bogdanowicz, R. G. Harrison, B. S. Coates, and E. B. Dopman. 2017. A combination of sexual and ecological divergence contributes to rearrangement spread during initial stages of speciation. Molecular Ecology 26:23312347. 
Lucas, L. K., C. C. Nice, and Z. Gompert. 2018. Genetic constraints on wing pattern variation in Lycaeides butterflies: A case study on mapping complex, multifaceted traits in structured populations. Molecular Ecology Resources 18:892-907.

Lucek, K., Z. Gompert, and P. Nosil. 2019. The role of structural genomic variants in population differentiation and ecotype formation in Timema cristinae walking sticks. Molecular Ecology 28:1224-1237.

Lynch, M., and A. Force. 2000. The probability of duplicate gene preservation by subfunctionalization. Genetics 154:459-473.

Lynch, M., M. O’Hely, B. Walsh, and A. Force. 2001. The probability of preservation of a newly arisen gene duplicate. Genetics 159:1789-1804.

Mahmoud, M., N. Gobet, D. I. Cruz-Dávalos, N. Mounier, C. Dessimoz, and F. J. Sedlazeck. 2019. Structural variant calling: the long and the short of it. Genome Biology 20:246.

Martin, S. H., J. W. Davey, C. Salazar, and C. D. Jiggins. 2019. Recombination rate variation shapes barriers to introgression across butterfly genomes. PLOS Biology 17:e2006288.

Masly, J. P., and D. C. Presgraves. 2007. High-resolution genome-wide dissection of the two rules of speciation in Drosophila. PLOS Biology 5:e243.

Montgomery, S. B., D. L. Goode, E. Kvikstad, C. A. Albers, Z. D. Zhang, X. J. Mu, G. Ananda, Genomes Project Consortium, D. G. MacArthur, A. Sidow, L. Duret, M. Gerstein, K. D. functional impact of short insertion-deletion variants identified in 179 human genomes. Genome Research 23:749-761.

Nosil, P. 2012. Ecological Speciation. Oxford University Press.
Nosil, P., J. L. Feder, S. M. Flaxman, and Z. Gompert. 2017. Tippi
speciation. Nature Ecology and Evolution 1:1-8.

Nosil, P., J. L. Feder, S. M. Flaxman, and Z. Gompert. 2017. Tipping points in the dynamics of

Nosil, P. 2012. Ecological Speciation. Oxford University
Nosil, P., J. L. Feder, S. M. Flaxman, and Z. Gompert. 2
speciation. Nature Ecology and Evolution 1:1-8.

Noor, M. A. F., K. L. Grams, L. A. Bertucci, and J. Reiland. 2001. Chromosomal inversions and the reproductive isolation of species. Proceedings of National Academy of Sciences of the United States of America 98:12084-12088.

Nosil, P., J. L. Feder, and Z. Gompert. 2021. How many genetic changes create new species? Science 371:777-779. 
Ohta, T. 1971. Associative overdominance caused by linked detrimental mutations. Genetics Research 18(3), 277-286.

Parchman, T. L., Z. Gompert, M. J. Braun, R. T. Brumfield, D. B. McDonald, J. a. C. Uy, G. Zhang, E. D. Jarvis, B. A. Schlinger, and C. A. Buerkle. 2013. The genomic consequences of adaptive divergence and reproductive isolation between species of manakins. Molecular Ecology 22:3304-3317.

Payseur, B. A. 2010. Using differential introgression in hybrid zones to identify genomic regions involved in speciation. Molecular Ecology Resources 10:806-820.

Payseur, B. A., J. G. Krenz, and M. W. Nachman. 2004. Differential patterns of introgression

Payseur, B. A., and L. H. Rieseberg. 2016. A genomic perspective on hybridization and speciation. Molecular Ecology 25:2337-2360.

Pritchard, J. K., M. Stephens, and P. Donnelly. 2000. Inference of population structure using multilocus genotype data. Genetics 155:945-959.

Ren, J., and M. J. P. Chaisson. 2021. lra: A long read aligner for sequences and contigs. PLOS Computational Biology 17:e1009078.

Rieseberg, L. H. 2001. Chromosomal rearrangements and speciation. Trends in Ecology \& Evolution 16:351-358.

Schumer, M., C. Xu, D. L. Powell, A. Durvasula, L. Skov, C. Holland, J. C. Blazier, S. Sankararaman, P. Andolfatto, G. G. Rosenthal, and M. Przeworski. 2018. Natural selection interacts with recombination to shape the evolution of hybrid genomes. Science 360:656-660.

Scott, J. A. 1992. The butterflies of North America: A natural history and field guide. Stanford University Press.

Sedlazeck, F. J., P. Rescheneder, M. Smolka, H. Fang, M. Nattestad, A. von Haeseler, and M. C. Schatz. 2018. Accurate detection of complex structural variations using single molecule sequencing. Nature Methods 15:461-468. Peichel, G.-P. Saetre, C. Bank, Å. Brännström, A. Brelsford, C. S. Clarkson, F. Eroukhmanoff, J. L. Feder, M. C. Fischer, A. D. Foote, P. Franchini, C. D. Jiggins, F. C. 
Jones, A. K. Lindholm, K. Lucek, M. E. Maan, D. A. Marques, S. H. Martin, B. Matthews, J. I. Meier, M. Möst, M. W. Nachman, E. Nonaka, D. J. Rennison, J. Schwarzer, E. T. Watson, A. M. Westram, and A. Widmer. 2014. Genomics and the origin of species. Nat Rev Genet 15:176-192.

Semenov, G. A., E. Linck, E. D. Enbody, R. B. Harris, D. R. Khaydarov, P. Alström, L. architecture of a plumage trait. Nature Communication 12:1019.

Serrato-Capuchina, A., and D. R. Matute. 2018. The Role of Transposable Elements in Speciation. Genes 9:254.

Servedio, M. R., G. S. V. Doorn, M. Kopp, A. M. Frame, and P. Nosil. 2011. Magic traits in speciation: 'magic' but not rare? Trends in Ecology \& Evolution 26:389-397.

Shastry, V., P. E. Adams, D. Lindtke, E. G. Mandeville, T. L. Parchman, Z. Gompert, and C. A. Buerkle. 2021. Model-based genotype and ancestry estimation for potential hybrids with mixed-ploidy. Molecular Ecology Resources 21:1434-1451.

Smadja, C. M., and R. K. Butlin. 2011. A framework for comparing processes of speciation in the presence of gene flow. Molecular Ecology 20:5123-5140.

Stebbins, G. L. 1958. The inviability, weakness, and sterility of interspecific hybrids. Pp. 147215 in M. Demerec, ed. Advances in Genetics. Academic Press.

Teeter, K. C., L. M. Thibodeau, Z. Gompert, C. A. Buerkle, M. W. Nachman, and P. K. Tucker. 2010. The variable genomic architecture of isolation between hybridizing species of house mice. Evolution 64:472-485.

Thompson, K. A., C. L. Peichel, D. J. Rennison, M. D. McGee, A. Y. K. Albert, T. H. Vines, A.

van Dijk, E. L., Y. Jaszczyszyn, D. Naquin, and C. Thermes. 2018. The third revolution in sequencing technology. Trends in Genetics 34:666-681.

794 Vila, R., C. D. Bell, R. Macniven, B. Goldman-Huertas, R. H. Ree, C. R. Marshall, Z. Bálint, K. 795 Johnson, D. Benyamini, and N. E. Pierce. 2011. Phylogeny and palaeoecology of Polyommatus blue butterflies show Beringia was a climate-regulated gateway to the New World. Proceedings of the Royal Society B: Biological Sciences 278:2737-2744. 
Villoutreix, R., C. F. de Carvalho, V. Soria-Carrasco, D. Lindtke, M. De-la-Mora, M. Muschick, J. L. Feder, T. L. Parchman, Z. Gompert, and P. Nosil. 2020. Large-scale mutation in the evolution of a gene complex for cryptic coloration. Science 369:460-466.

Wagner, D. N., R. L. Curry, N. Chen, I. J. Lovette, and S. A. Taylor. 2020. Genomic regions underlying metabolic and neuronal signaling pathways are temporally consistent in a moving avian hybrid zone. Evolution 74:1498-1513.

Weissensteiner, M. H., I. Bunikis, A. Catalán, K.-J. Francoijs, U. Knief, W. Heim, V. Peona, S. D. Pophaly, F. J. Sedlazeck, A. Suh, V. M. Warmuth, and J. B. W. Wolf. 2020. Discovery and population genomics of structural variation in a songbird genus. Nature Communications 11:3403.

Wright, S. 1978. Modes of Speciation. Michael J. D. White W. H. Freeman and Co., San Francisco. 1978. VIII + 456 pp. illus. \$27.50. Paleobiology 4:373-379. Cambridge University Press.

Yeaman, S. 2013. Genomic rearrangements and the evolution of clusters of locally adaptive loci.

817 Zhang, L., R. Reifová, Z. Halenková, and Z. Gompert. 2021a. How important are structural

Zhang, L., G. R. Hood, I. Carroo, J. R. Ott, and S. P. Egan. 2021b. Context-Dependent Reproductive Isolation: Host Plant Variability Drives Fitness of Hybrid Herbivores. The American Naturalist 197:732-739

Zhou, Y. 2019. The population genetics of structural variants in grapevine domestication. Nature Plants 5:17.

Zuellig, M. P., and A. L. Sweigart. 2018. Gene duplicates cause hybrid lethality between 
829 Table 1. Number and length (mean, 2.5th and 97.5th percentiles) of SVs detected by nanopore

830 sequencing; $\mathrm{SV}$ types: $\mathrm{INV}=$ inversion, $\mathrm{DUP}=$ duplication, $\mathrm{INS}=$ insertion, and $\mathrm{DEL}=$

831 deletion.

\begin{tabular}{llllll}
\hline & \multirow{2}{*}{ SV type } & \multirow{2}{*}{ No. SVs } & \multicolumn{3}{c}{ Length of SVs (bp) } \\
& & & Mean & $\mathbf{2 . 5 0 \%}$ & $\mathbf{9 7 . 5 0 \%}$ \\
\hline \multirow{3}{*}{ All SVs } & DEL & 68927 & 1571 & 42 & 11554 \\
& DUP & 617 & 5472 & 51 & 20393 \\
& INS & 56527 & 359 & 43 & 803 \\
& INV & 1503 & 41306 & 175 & 422305 \\
\hline \multirow{3}{*}{ SVs with } & DEL & 13359 & 7171 & 1035 & 28073 \\
length>1000 bp & DUP & 116 & 27697 & 1074 & 356753 \\
& INS & 771 & 8751 & 1000 & 42286 \\
& INV & 1073 & 57670 & 1104 & 542297 \\
\hline
\end{tabular}

833

834 Table 2. SVs detected by mate-pair sequencing and number of mate-pair SVs that overlapped with

835 those found by nanopore sequencing; SV types: INV = inversion, DUP = duplication, INS = 836 insertion, and DEL = deletion.

\begin{tabular}{|c|c|c|c|c|c|}
\hline \multirow{2}{*}{ SV type } & \multirow{2}{*}{ No. SVs } & \multicolumn{3}{|c|}{ Length of SV (bp) } & \multirow{2}{*}{$\begin{array}{l}\text { No. SVs } \\
\text { overlapped }\end{array}$} \\
\hline & & Mean & $2.50 \%$ & $97.50 \%$ & \\
\hline DEL & 11594 & 4771 & 35 & 7222 & 3862 \\
\hline DUP & 224 & 82866 & 125 & 810257 & 14 \\
\hline INV & 156 & 270236 & 97 & 1748269 & 15 \\
\hline Total & 11974 & & & & 3891 \\
\hline
\end{tabular}


842 Table 3. Comparison of pairwise Fst across different structural variant types. Lineage codes: JH

$843=$ Jackson Hole Lycaeides, LM = L. melissa, DBS = Dubois hybrid zone. SV types: INV =

844 inversion, DUP = duplication, INS = insertion, and DEL = deletion.

\begin{tabular}{llllll}
\hline Pairs & All SVs & INV & DUP & INS & DEL \\
\hline JH-LM & 0.048 & 0.07 & 0.044 & 0.047 & 0.048 \\
\hline DBS-JH & 0.022 & 0.035 & 0.025 & 0.021 & 0.023 \\
\hline DBS-LM & 0.03 & 0.043 & 0.028 & 0.031 & 0.029 \\
\hline
\end{tabular}

850 Table 4. Results of multiple linear regression models of FST against length and number of genes

851 within each SV. SV types: INV = inversion, DUP = duplication, INS = insertion, and DEL = 852 deletion.

\begin{tabular}{llrcrr}
\hline SV type & Factor & Estimate & SE of estimate & $\boldsymbol{t}$-value & $\begin{array}{r}\boldsymbol{P} \text { - } \\
\text { value }\end{array}$ \\
\hline \multirow{3}{*}{ DEL } & length & 0.094 & 0.023 & 4.103 & $<\mathbf{0 . 0 0 1}$ \\
& number of genes & 0.055 & 0.024 & 2.242 & $\mathbf{0 . 0 2 5}$ \\
& length: no. genes & -0.004 & 0.001 & -5.345 & $<\mathbf{0 . 0 0 1}$ \\
\hline \multirow{3}{*}{ DUP } & length & -0.009 & 0.103 & -0.090 & 0.928 \\
& number of genes & -0.007 & 0.100 & -0.068 & 0.946 \\
& length: no. genes & 0.001 & 0.003 & 0.425 & 0.671 \\
\hline \multirow{3}{*}{ INS } & length & -0.066 & 0.066 & -0.994 & 0.320 \\
& number of genes & -0.030 & 0.044 & -0.682 & 0.495 \\
& length: no. genes & 0.004 & 0.002 & 1.502 & 0.133 \\
\hline \multirow{3}{*}{ INV } & length & 0.019 & 0.017 & 1.111 & 0.267 \\
& number of genes & 0.009 & 0.025 & 0.378 & 0.705 \\
& length: no. genes & -0.001 & 0.001 & -1.142 & 0.254 \\
\hline
\end{tabular}


855 Table 5. Results of multiple linear regression models of $\alpha$ against length and number of genes

856 within each SV. SV types: INV = inversion, DUP = duplication, INS = insertion, and DEL = 857 deletion.

\begin{tabular}{clcccc}
\hline \multirow{2}{*}{ SV type } & Factor & Estimate & SE of estimate & $\boldsymbol{t}$-value & $\begin{array}{c}\boldsymbol{P} \text { - } \\
\text { value }\end{array}$ \\
\hline \multirow{2}{*}{ DEL } & length & 0.248 & 0.082 & 3.023 & $\mathbf{0 . 0 0 3}$ \\
& number of genes & 0.016 & 0.110 & 0.148 & 0.883 \\
& length: no. genes & -0.029 & 0.012 & -2.422 & $\mathbf{0 . 0 1 6}$ \\
\hline \multirow{2}{*}{ DUP } & length & 0.175 & 1.388 & 0.126 & 0.906 \\
& number of genes & -0.841 & 1.132 & -0.743 & 0.499 \\
& length: no. genes & 1.321 & 1.929 & 0.685 & 0.531 \\
\hline \multirow{2}{*}{ INS } & length & -0.171 & 0.520 & -0.328 & 0.745 \\
& number of genes & -0.987 & 1.786 & -0.552 & 0.585 \\
& length: no. genes & 0.105 & 0.135 & 0.779 & 0.443 \\
\hline \multirow{2}{*}{ INV } & length & -0.047 & 0.060 & -0.789 & 0.431 \\
& number of genes & 0.185 & 0.079 & 2.348 & $\mathbf{0 . 0 2 1}$ \\
& length: no. genes & -0.010 & 0.006 & -1.578 & 0.117 \\
\hline
\end{tabular}



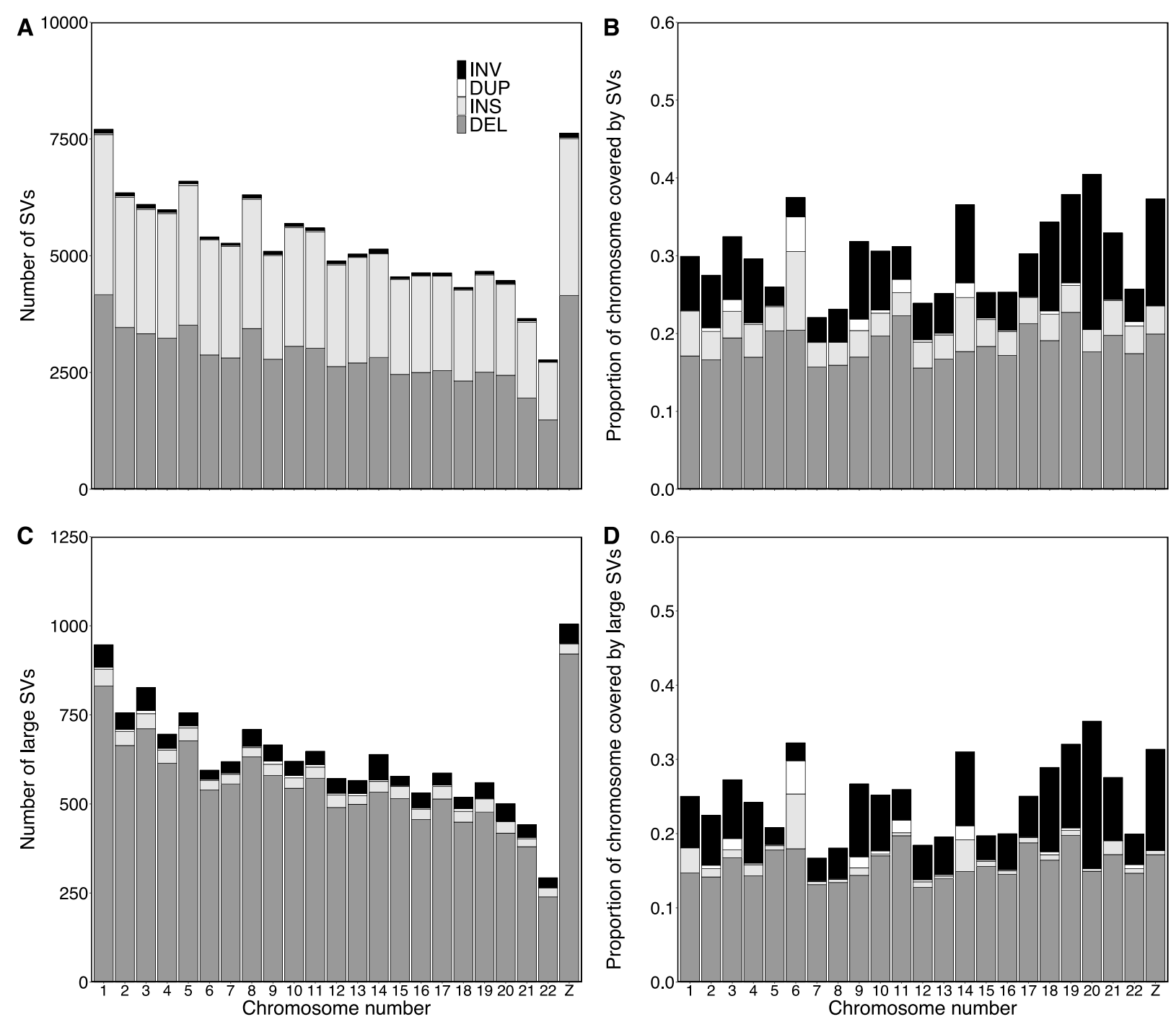

Figure 1. (A) Number of structural variants (SVs) across the genome. (B) Proportion of each chromosome covered by SVs. (C) Number of large SVs (>1000 bp) across the genome. (D) Proportion of each chromosome covered by large SVs (>1000 bp). Bars with different colors denote different SV types, as shown in the legend: INV = inversion, DUP = duplication, INS = insertion, and DEL = deletion. 

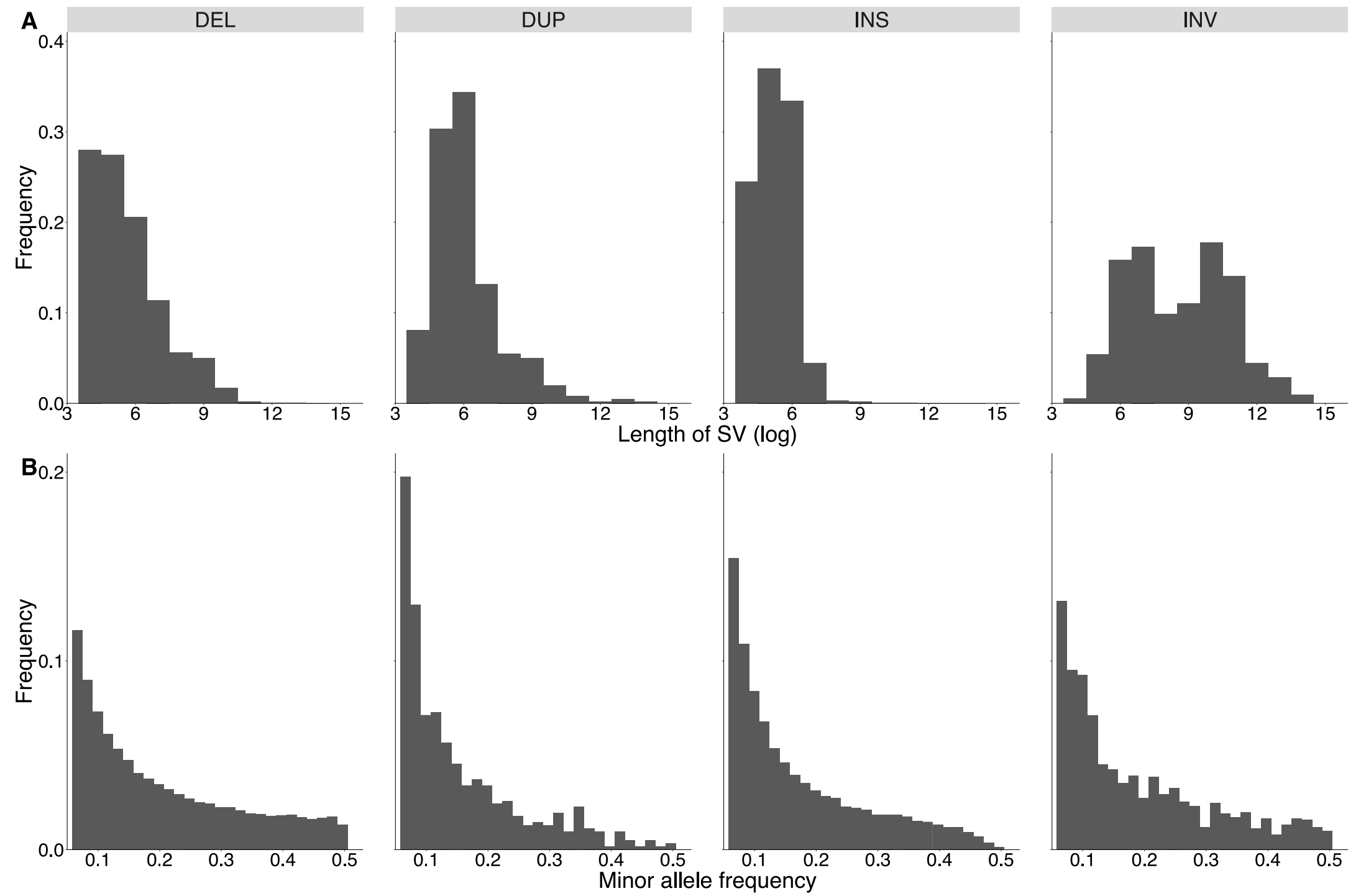

Figure 2. Histograms of distribution of (A) length of SV (log) across different types of structural variants, (B) minor allele frequency of all 39 individuals of Lycaeides. 
(A)

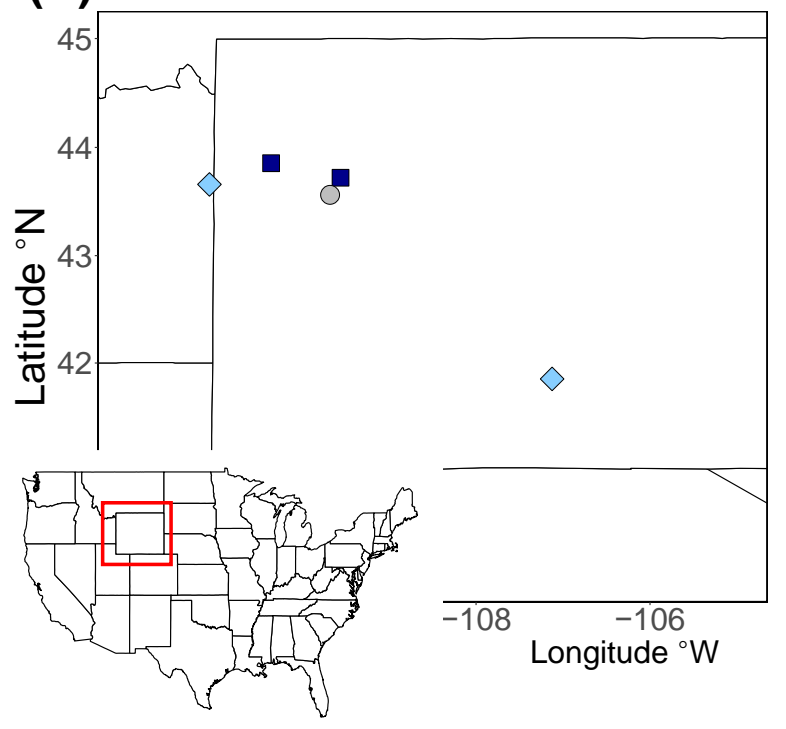

(B)

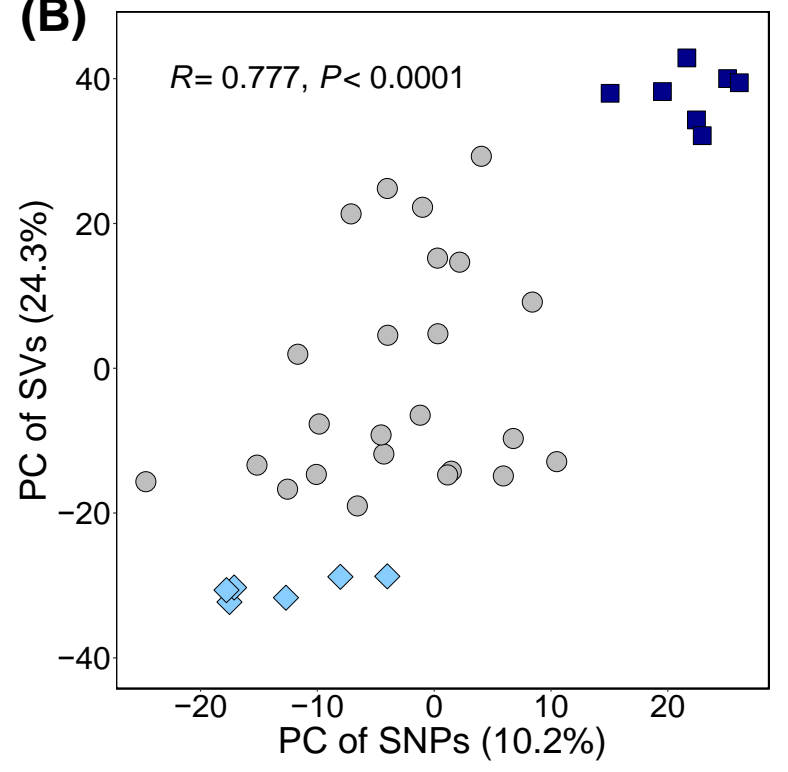

(C)

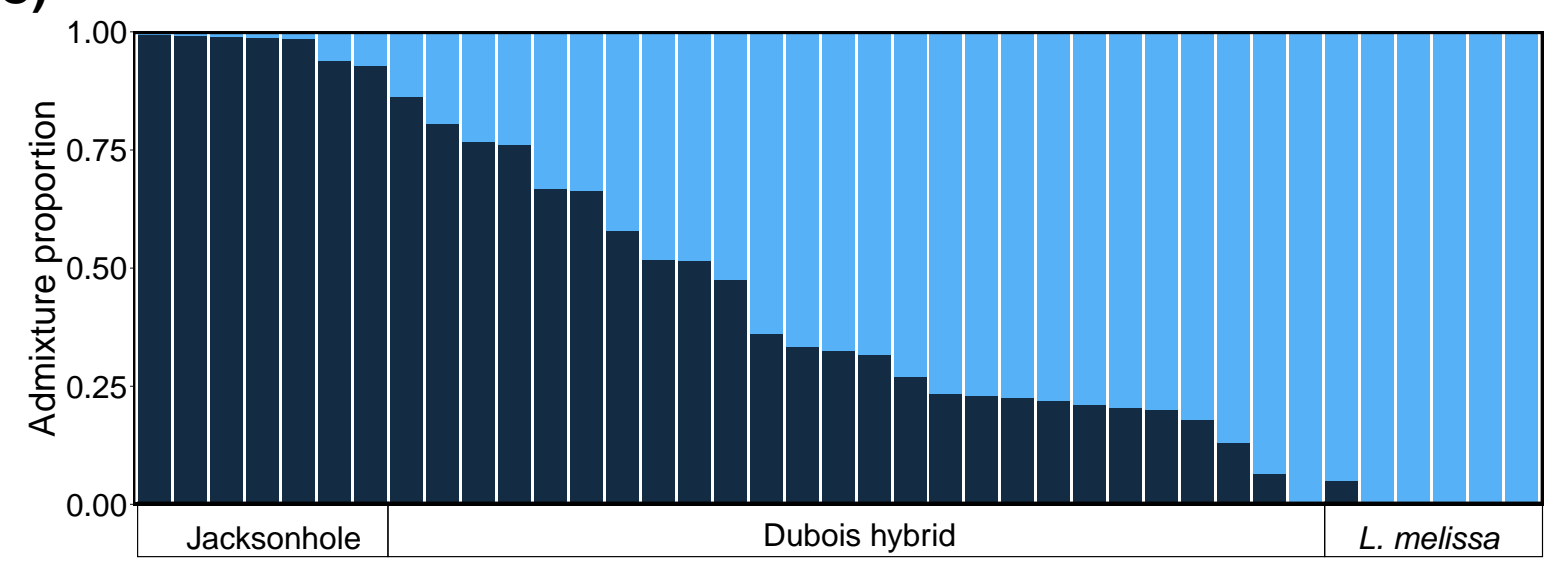

Figure 3. (A) Collection sites, (B) Plot of PC score 1 of large, ancestry informative SVs (>1000bp, allele frequency differences $>0.3$ ) against PC score 2 of SNPs, (C) Entropy plot from large, ancestry informative SVs (>1000bp, allele frequency differences $>0.3)$, y axis is the Bayesian estimates of admixture proportion. 


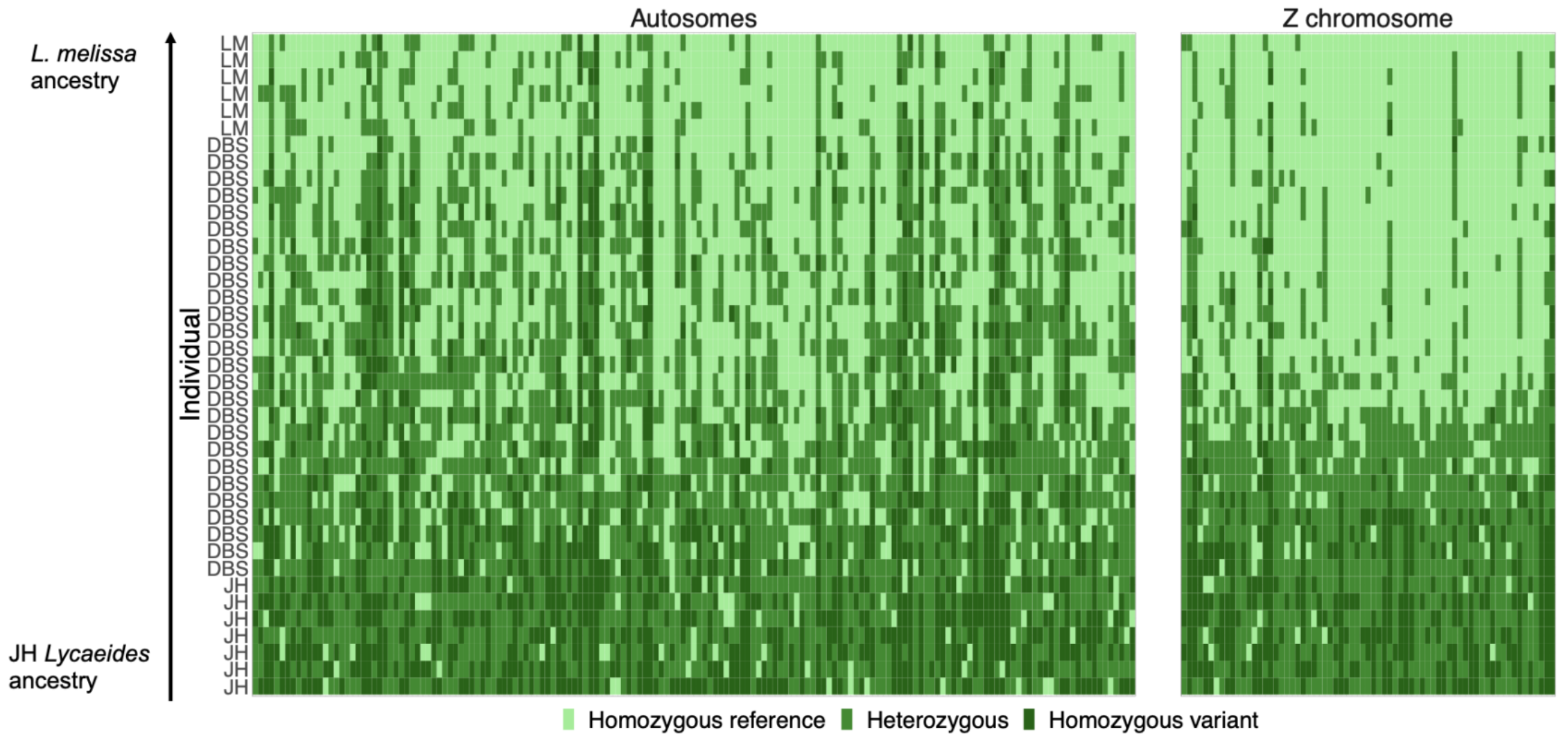

Figure 4. Genotypes of ancestry informative loci (allele frequency differences $>0.5$ ) on autosomes vs. the $\mathrm{Z}$ chromosome across individual butterflies. Each colored rectangle denotes the genotype estimate for a SV locus (x-axis) and individual (y-axis). Genotype estimates were rounded to the nearest whole number for visualization. Loci on the $\mathrm{x}$-axis are ordered by first chromosome number, then by position of the locus, and lastly by types of SVs and length. Individuals are sorted by the order of the hybrid index (proportion of ancestry from L. melissa). JH stands for parental species Jackson Hole Lycaeides, DBS stands for Dubois hybrid individuals, LM stands for parental species L. melissa. 

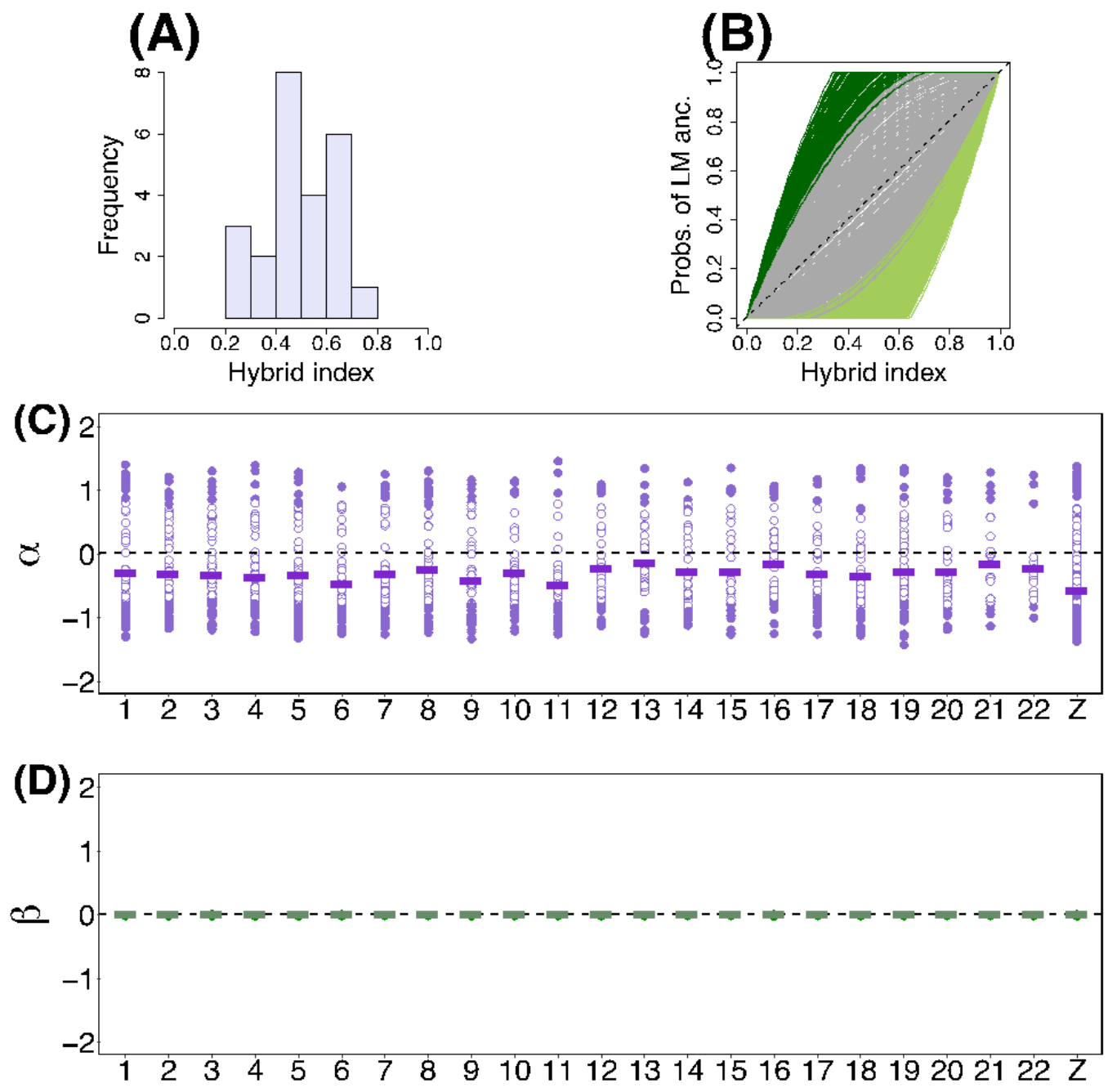

Figure 5. Summary of the genomic cline analysis. (A) Distribution of hybrid index using structural variant data. (B) Estimated genomic cline relative to SNPs using ancestry-informative SVs. Each solid line gives the estimated probability of Jackson Hole $(\mathrm{JH})$ ancestry for a structural variant locus. Green lines denote cases of credible directional introgression (95\% ETPIs for $\alpha$ that do not overlap with zero), gray lines denote clines not significantly different from the genome average. The dashed line gives the null expectation based on genome-wide admixture. Distribution of cline parameters $\alpha(\mathbf{C})$ and $\beta(\mathbf{D})$ across different chromosomes. Solid circles indicate loci with cline parameters indicating credible deviations from genome-average introgression (95\% ETPIs that do not overlap with zero), whereas unfilled circles indicate loci not significantly different from the genome average. Solid horizontal lines are the mean value of cline parameters across all loci at each chromosome. The dashed horizontal line gives the null expectation based on genome-wide admixture using SNP loci. 
(A)

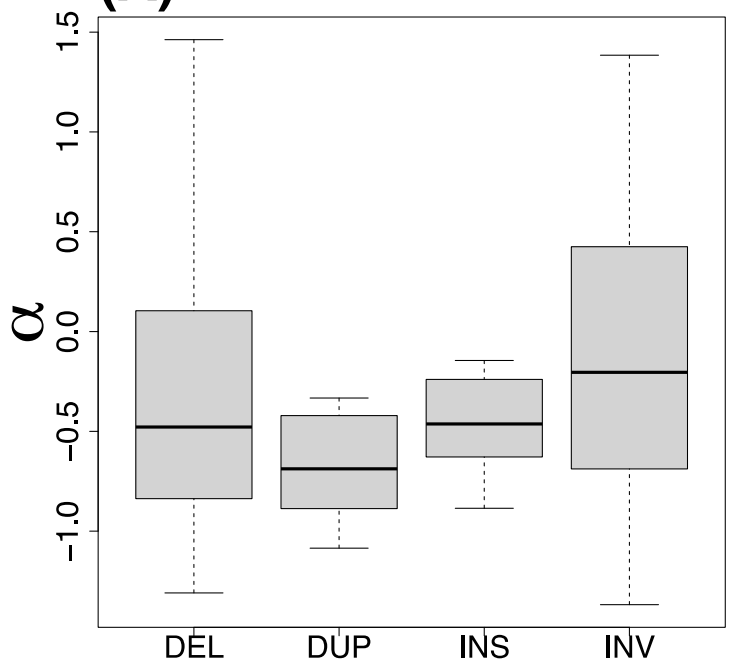

Types of structural variants

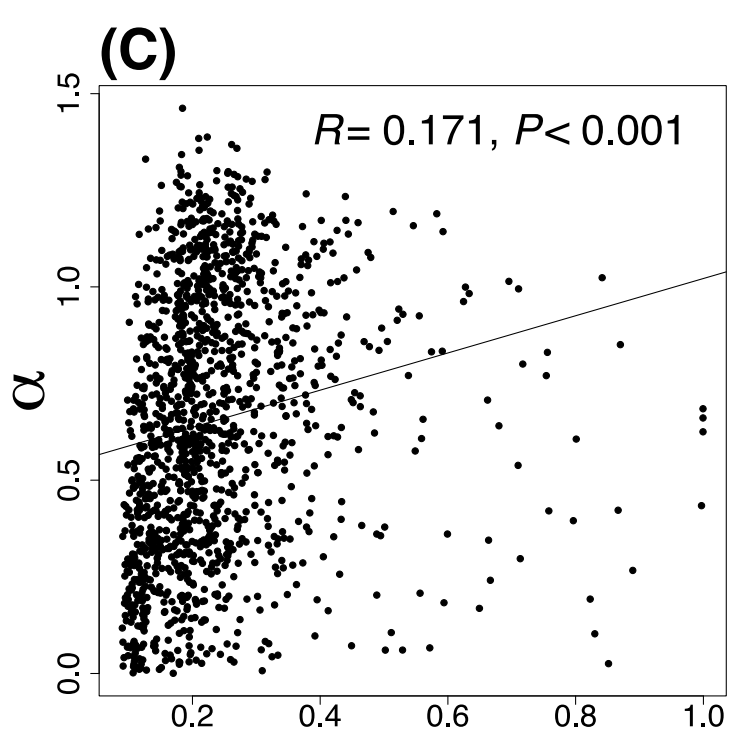

Fst
(B)

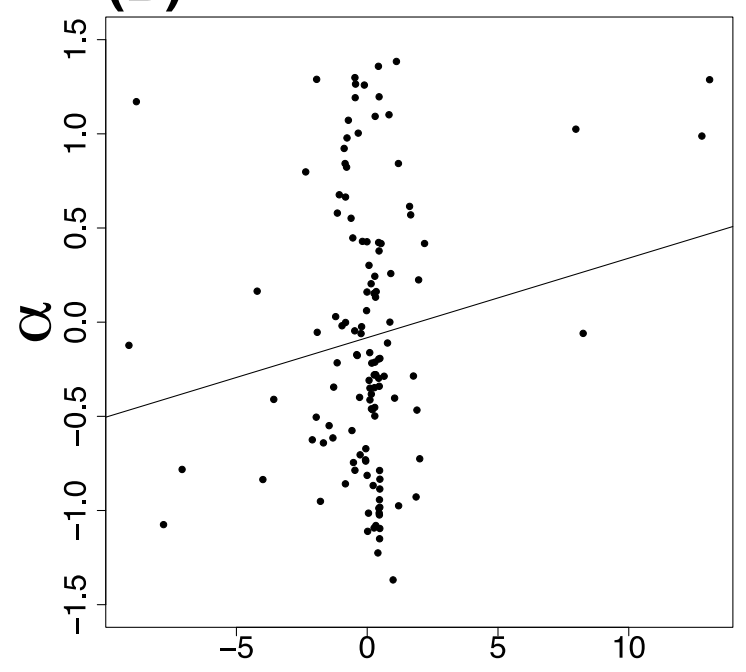

Residuals of the number of genes against length

Figure 6. (A) Comparison of cline parameter $\alpha$ across different structural variant types. (B) Correlation between $\alpha$ and the residuals of the number of genes against the length across all inversions. (C) Correlation between $\alpha$ and FST across loci. 